\title{
The role of narrow-angle forward surface scatter and particulate scatter in exoplanet exploration
}

Richard N. Pfisterer, James E. Harvey, James B. Breckinridge

Richard N. Pfisterer, James E. Harvey, James B. Breckinridge, "The role of narrow-angle forward surface scatter and particulate scatter in exoplanet exploration," Proc. SPIE 10698, Space Telescopes and Instrumentation 2018: Optical, Infrared, and Millimeter Wave, 106985F (6 July 2018); doi: $10.1117 / 12.2314229$

SPIE Event: SPIE Astronomical Telescopes + Instrumentation, 2018, Austin, Texas, United States 


\title{
The Role of Narrow-angle Forward Surface Scatter and Particulate Scatter in Exoplanet Exploration
}

\author{
Richard N. Pfisterer ${ }^{\mathrm{a}}$, James E. Harvey ${ }^{\mathrm{a}}$ and James B. Breckinridge ${ }^{\mathrm{b}}$ \\ ${ }^{a}$ Photon Engineering, LLC, 310 S. Williams Blvd., Suite 222, Tucson, AZ 85711 \\ ${ }^{\mathrm{b}}$ Breckinridge Associates, LLC and California Institute of Technology, Pasadena, CA
}

\begin{abstract}
It is often thought that because of the very small solid angle subtended by the field-of-view of the coronagraph, scattered light from optical surfaces will have no effect on images recorded for terrestrial exoplanet spectroscopy. In this paper, we examine mirror surface scatter and particulate contamination scatter as sources of background light or noise signal in large aperture terrestrial exoplanet telescope/coronagraph systems. Scattered light control to one part in $10^{+10}$ or better is required for exoplanet exploration. We will discuss the optical fabrication tolerances necessary to minimize narrowangle forward scatter and their relative effects upon direct imaging coronagraph instruments used to characterize terrestrial exoplanets.
\end{abstract}

Keywords: stray light, BSDF, particulates, exoplanets

\section{INTRODUCTION}

It is obvious from photographs taken with large telescopes that narrow-angle forward surface scatter and particulate scatter are detrimental to exoplanet detection as shown in Figure 1. These scatter mechanisms are most significant in the vicinity of the star, which is exactly where we'd expect to see a dim planet in orbit.

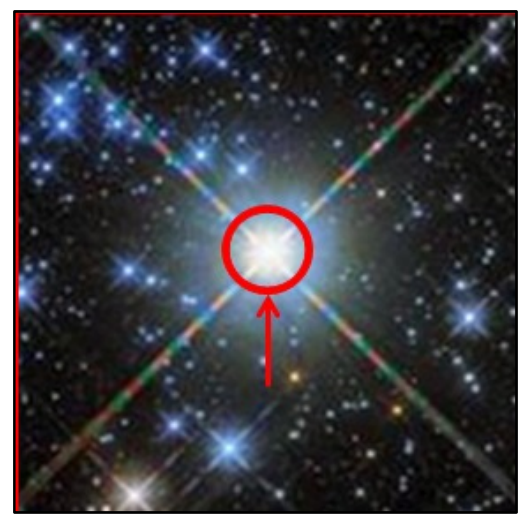

Figure 1 Photograph taken with Hubble Space Telescope showing diffraction and scatter effects in the vicinity of a star. These make the detection of exoplanets a very challenging problem.

While rigorous stray light analyses require the development of complex models to be run on specialized software, it is possible, using straightforward radiometric theory, to derive the equations for the scatter contributions at the detector of a Cassegrain telescope (the most conventional telescope configuration) from both mirror surface roughness and particulate contamination. 


\section{STELLAR RADIOMETRY}

Since our goal is to derive expressions that can be manipulated easily to gain insight, we can start with a whole series of simplifying assumptions regarding the radiometry of the star and planets:

We assume that all stars (and planets) are of the same spectral class as the sun. Technically this is unnecessary but it does make the calculations simpler because we don't have to take the spectra into account.

We assume that the sun has an apparent stellar magnitude of -26.7. If the star's apparent stellar magnitude is $m_{\text {star }}$, then the ratio of irradiances (power/area) at the telescope's primary mirror (entrance aperture) is given by

$$
\text { Ratio }_{E}=2.512^{\left(-26.7-m_{\text {star }}\right)}
$$

We assume that the telescope is exoatmospheric to avoid having to calculate the transmission through the atmosphere.

In order to get the relative irradiance scaling between the sun and the star correct, we assume that the solar constant is $1366 \mathrm{w} / \mathrm{m}^{2}$ for an infinite spectral bandwidth. However detectors do not have an infinite spectral bandwidth and so we need to impose a bandwidth on the calculations. The fractional spectral bandwidth $f$ is the ratio of the integration of the radiance over the spectral bandwidth (from a minimum wavelength $\lambda_{1}$ to a maximum wavelength $\lambda_{2}$ ) to the infinite spectral bandwidth integral:

$$
f\left(\lambda_{1}, \lambda_{2}, T\right)=\int_{\lambda_{1}}^{\lambda_{2}} L_{\lambda}(\lambda, T) d \lambda / \int_{0}^{\infty} L_{\lambda}(\lambda, T) d \lambda
$$

Finally the star's irradiance at the telescope aperture is given by

$$
E_{\text {pristar }}=\text { Ratio }_{E} \cdot 1366 \cdot f \quad\left[\frac{\text { watts }}{m^{2}}\right]
$$

We can readily calculate the irradiance of a star (or planet) on the detector knowing the incident irradiance on the entrance aperture of the telescope. From aberration theory, the area of the core of the Airy function (that contains $83.9 \%$ of the light) for a diffraction-limited system is given by

$$
\frac{\pi}{4} \cdot\left(2.44 \cdot \lambda \cdot \text { fnumber }^{2}\right.
$$

where $\lambda$ is the wavelength of the incident light and fnumber is the ratio of the effective focal length to the entrance aperture diameter. Combining equations (3) and (4), and including the transmission losses $\tau$, the irradiance of a star (or planet) at the detector is therefore given by

$$
E_{\text {det }, \text { star }}=\frac{0.84 \cdot \tau \cdot E_{\text {pri,star }} \cdot D^{2}}{(2.44 \cdot \lambda \cdot \text { fnumber })^{2}}
$$

where $D$ is the diameter of the entrance aperture. 


\subsection{Surface Microroughness Scatter}

\section{SCATTER THEORY}

The Harvey-Shack BSDF model [1] is commonly used to describe the near-specular scatter produced by a "smooth" optical surface, i.e., a surface whose rms roughness $\sigma_{\mathrm{rms}}$ is much less than the wavelength of the incident light.

Fortunately this is a reasonable assumption for virtually all visible and IR telescopes.

The Harvey-Shack BSDF model can be written in numerous forms; for the sake of simplicity we use a functional form that is parameterized by three variables: $b_{0}, S$, and $L$, viz.

$$
\operatorname{BSDF}\left(\beta-\beta_{0}\right)=b_{0}\left[1+\left(\frac{\beta-\beta_{0}}{L}\right)^{2}\right]^{\frac{S}{2}}
$$

where $\beta-\beta_{0}=\sin ($ scattering angle) $-\sin ($ specular angle). However since the telescope will be planet-hunting near the center of the field, we can assume that the specular angle is zero and therefore write a simpler expression for the BSDF:

$$
\operatorname{BSDF}(\beta)=b_{0}\left[1+\left(\frac{\beta}{L}\right)^{2}\right]^{S / 2}
$$

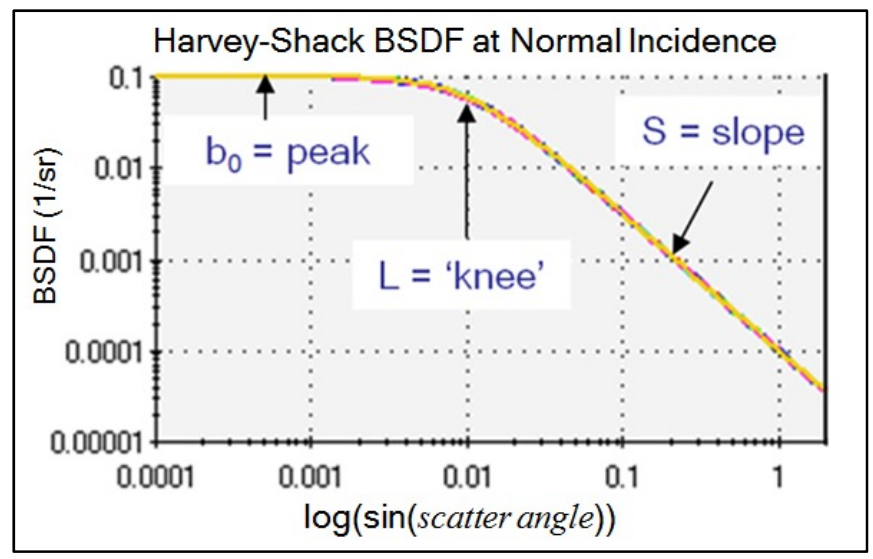

Figure 2 Parametric variables that describe the Harvey-Shack scatter function for smooth optical surfaces.

The physical significance of each parameter is as follows:

The $b_{o}$ parameter is the near-specular (peak) BSDF value and is related to $\sigma_{r m s}$ by

$$
\sigma_{r m s}^{2}= \begin{cases}\left(\frac{\lambda}{2 \Delta n}\right)^{2} \frac{b_{o}}{\pi} L^{2} \log _{e}\left(1+\frac{1}{L^{2}}\right) & \text { for } S=-2 \\ \left(\frac{\lambda}{\Delta n}\right)^{2} \frac{b_{o}}{2 \pi L^{S}(S+2)}\left[\left(1+L^{2}\right)^{\frac{S+2}{2}}-\left(L^{2}\right)^{\frac{S+2}{2}}\right] & \text { for } S \neq-2\end{cases}
$$


Note that the exact specular direction is not shown in Figure 2 because the abscissa is plotted in log space.

The $S$ parameter is related to the power-law falloff of the surface power spectral density (PSD) function. Typically $S$ is approximately -2, although with high-speed computer-controlled grinding/polishing we have observed $S$ as low as -2.9. (While seemingly unrelated, there is a complex relationship between the $S$ and $b_{0}$ parameters and $\sigma_{r m s}$. The $S$ parameter is the power-law falloff of the 2D PSD, the integral of the 2D PSD is equal to $\sigma_{r m s}{ }^{2}$, and as shown in equation $7, \sigma_{r m s}{ }^{2}$ is proportional to $b_{0}$. Consequently for a given $\sigma_{r m s}$, a large negative value for $S$ corresponds to a large value for $b_{0} \ldots$ exactly the opposite of what is desirable for planetary detection from a scatter standpoint!)

The $L$ parameter is the angle at which the BSDF transitions from a power-law falloff to a constant Lambertian value. Since an accurate measurement of $L$ is experimentally difficult due to instrument signature and diffraction issues, theorists have proposed that "reasonable" values for $L$ (when L cannot be measured) lie in the range of 0.0001 to 0.01 radians (20.6 to 2063 arc-seconds) from specular. We will be searching for planets at angles closer to specular than this and so it is reasonable to assume that the BSDF is constant for this application.

\subsection{Particulate Scatter}

Since it is statistically unstable to ray trace individual particulates on a surface, stray light analysts have developed and validated BSDF models for particulate distributions that can be run efficiently in software. Most of these models are based upon Mie theory which employs spherical particles. (Interestingly enough, "real" particles greater than 10 microns in diameter are rarely spherical but given the tremendous number of particles present on a surface and that they are oriented in random directions, a scatter model constructed from simple spherical particles generally describes the appropriate behavior.) Since Mie theory takes into account the complex refractive indices of the particles, models have been developed based upon measurements of dust particles in the air.

There are several ways to determine the particle size population: witness samples, automated particle diameter measurement devices, microscope analyses, etc. One commonly used estimate is defined by MIL-STD-1246 [2].

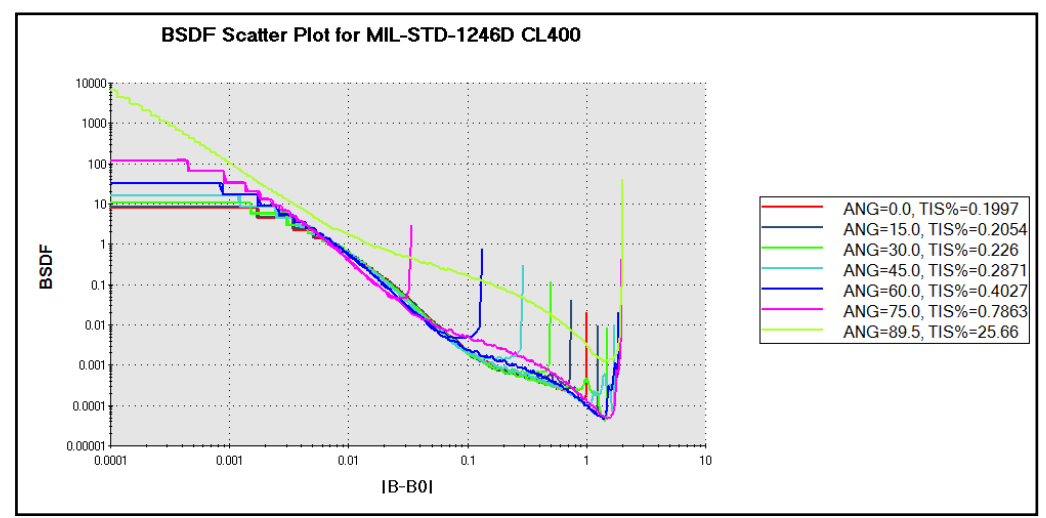

Figure 3 BSDF plot for MIL-STD-1246 CL400 particulate distribution in visible light for 7 angles of incidence.

The MIL-STD-1246 size population is given by

$$
\log _{10}\left(N_{a}\right)=0.926\left[\log _{10}^{2}(C L)-\log _{10}^{2}(x)\right]
$$

where $N_{a}$ is the number of particulates $/ 0.1 \mathrm{~m}^{2}$ greater than $x$ microns in diameter and $C L$ is the cleanliness level. 
While it is ubiquitous in aerospace applications, there are many problems with the MIL-STD-1246 particulate distribution:

- Actual particulate distributions do not follow this functional form.

- This distribution ignores particulates smaller than $1 \mu \mathrm{m}$ in diameter.

- Measurements of actual clean room fallouts at JPL, Northrup Grumman, Raytheon, etc. indicate that the more frequently observed slope for uncleaned surfaces is on the order of $0.303-0.557$ (average $=0.383$ ) [3].

However, despite these deficiencies, this distribution is in common use because of its simplicity and familiarity. Some authors have proposed changes intended to better model "real" particulate distributions [4].

Note that the BSDF due to particulates exhibits an inverse power law behavior very much like the BSDF for microroughness, despite the very different physical processes. Typically, the near-specular BSDF due to particulates is greater than that of surface roughness.

The conversion of a particulate size distribution function into a BSDF model that can be ray traced is not complex but involves several steps:

- The particulate diameter range divided into $N$ bins. (For numerical stability, the bins are established in $\log$ space.)

- For each bin:

- The average particulate diameter is calculated.

- The Mie intensity (phase) function for single particle/unit area with given complex refractive index at a specific wavelength is calculated.

- The Mie intensity function is multiplied by number of particulates in that bin.

- The Mie intensity functions are accumulated (summed).

The resulting Mie intensity function can be readily converted into a BSDF [5], viz.

$$
B S D F=\frac{\text { Mie intensity }}{\left(\frac{2 \pi}{\lambda}\right)^{2}}
$$

where $\lambda$ is the wavelength of the incident light. By evaluating a set of different populations and converting to BSDF, it is possible to establish a relationship between cleanliness level and $b_{0}$.

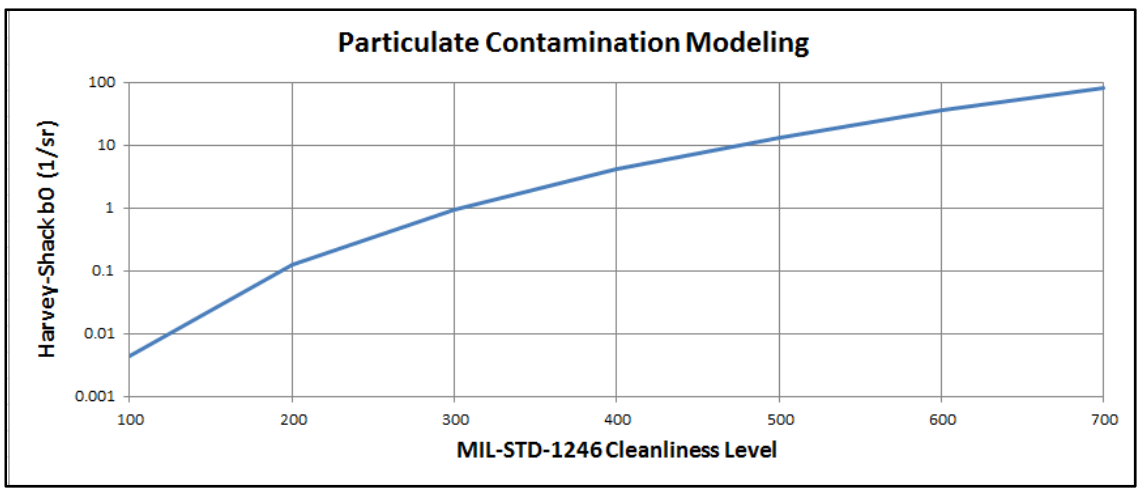

Figure 4 Derived relationship between Harvey-Shack $b_{0}$ parameter and MIL-STD-1246 cleanliness level. 


\section{TELESCOPE RADIOMETRY}

Starting with the fundamental theorem of radiometry and making the assumption that the telescope mirrors and detector share a common optical axis, we write

$$
d^{2} P_{c}=L_{s} \cdot d A_{s} \cdot d \Omega_{c}
$$

where $d^{2} P_{c}$ is the differential power collected by the collector, $L_{s}$ is the radiance of the source, $d A_{s}$ is the differential emitting area of the source, and $d \Omega_{c}$ is the projected solid angle of the collector. From this expression, we can readily derive the expression for the amount of power $P_{\text {detector }}$ scattered by a telescope mirror that is collected by the detector, viz.

$$
P_{\text {detector }}=P_{\text {mirror }} \cdot B S D F_{\text {mirror }} \cdot \Omega_{\text {detector }}
$$

where $P_{\text {mirror }}$ is the power incident on the mirror and $B S D F_{\text {mirror }}$ describes the surface scattering characteristics. Since we are dealing with small detectors and rather large telescopes, we can replace $\Omega_{\text {detector }}$ with the approximation

$$
\Omega_{\text {detector }} \approx \frac{A_{\text {detector }}}{r^{2}}
$$

where $A_{\text {detector }}$ is the area of the detector and $r$ is the optical distance from the scattering surface to the detector.

Since irradiance (power/area) is frequently a more meaningful metric in stray light analysis, we can rewrite the power expression in terms of irradiance $E_{\text {detector }}$

$$
E_{\text {detector }}=\frac{P_{\text {mirror }} \cdot B S D F_{\text {mirror }}}{r^{2}}
$$

Assuming that the telescope is a Cassegrain configuration with an aperture $D$ in diameter and an effective focal length efl, we can write the expressions for the scattered power from each mirror that will be collected by the detector

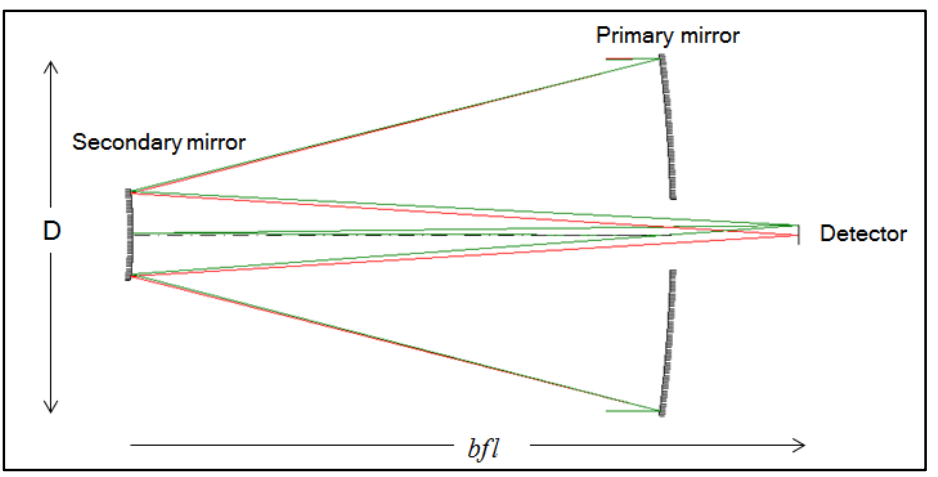

Figure 5 First-order layout of a Cassegrain Telescope

For the primary mirror:

$$
E_{\text {det }, p r i}=\frac{\tau \cdot P_{p r i} \cdot B S D F_{p r i}}{e f l^{2}}
$$


For the secondary mirror:

$$
E_{\text {det }, \mathrm{sec}}=\frac{P_{\mathrm{sec}} \cdot B S D F_{\text {sec }}}{b f l^{2}}
$$

where $\tau=$ telescope transmission and fnumber $=$ efl $/ D$.

It is reasonable to assume that the primary and secondary mirrors can have the same BSDF. To first order, the power incident on the secondary mirror is the same as the power incident on the primary mirror. Therefore the total scattered irradiances at the detector due to the primary and secondary mirrors are given by

$$
\begin{gathered}
E_{\text {det, }, \text { ri scatter }}=E_{\text {pri,star }} \cdot \tau \cdot \pi \cdot \frac{D^{2}}{4} \cdot \frac{b_{o, \text { rough }}+b_{o, \text { part }}}{(e f l)^{2}} \\
E_{\text {det, sec scatter }}=E_{\text {pri,star }} \cdot \pi \cdot \frac{D^{2}}{4} \cdot \frac{b_{o, \text { rough }}+b_{o, \text { part }}}{(b f l)^{2}}
\end{gathered}
$$

where $b_{o, \text { rough }}$ and $b_{o, \text { part }}$ are the BSDF $b_{o}$ parameters for surface roughness and particulates, respectively.

Since they are spatially incoherent, the total scattered irradiance on the detector is simply the sum of the individual mirror irradiance contributions

$$
E_{\text {det, scatter }}=E_{\text {det, } \text {, ri scatter }}+E_{\text {det, sec scatter }}
$$

\section{EXAMPLES}

Given the number of parameters that can be varied, there are an infinite number of cases that can be considered. We considered three examples based upon the following assumptions:

- $0^{\text {th }}, 6^{\text {th }}$ and $10^{\text {th }}$ magnitude star.

- $30^{\text {th }}$ magnitude planet.

- Telescope with the following parameters: $30 \mathrm{~m}$ diameter primary mirror working at $\mathrm{F} / 2,5 \mathrm{~m}$ diameter secondary mirror, $40 \mathrm{~m}$ primary-secondary mirror separation, $600 \mathrm{~m}$ effective focal length (F/20), $60 \mathrm{~m}$ back focal length

- Visible $(0.4-1.0 \mu \mathrm{m})$ spectral bandwidth.

- Exoatmospheric.

Note that the following plots show the image irradiance of the $30^{\text {th }}$ magnitude planet relative to the scattered irradiance of the star.

Figure 6 shows the detector irradiances for a system employing conventionally polished mirrors $(S=-2, L=0.01$ radians) contaminated with CL400 particulates ("visibly dirty") corresponding to typical "beginning of life" (BOL) in orbit. In this case, the planet is clearly visible against a $6^{\text {th }}$ or $10^{\text {th }}$ magnitude star, but nearly indistinguishable from the scatter produced by a $0^{\text {th }}$ magnitude star. 


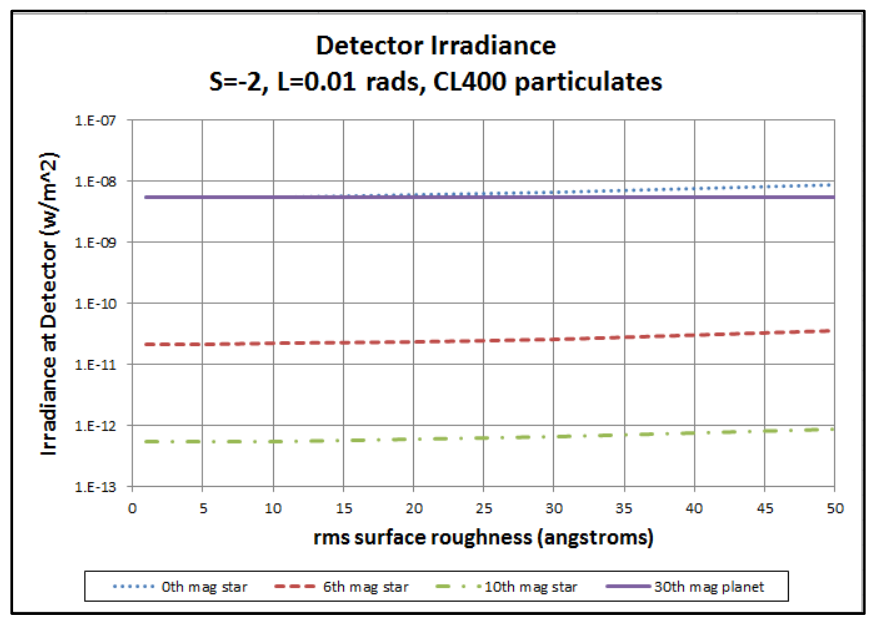

Figure 2 Detector irradiances for typical mirror surfaces and "visibly dirty" level of particulates (CL400, corresponding to "beginning of life" [BOL] in orbit).

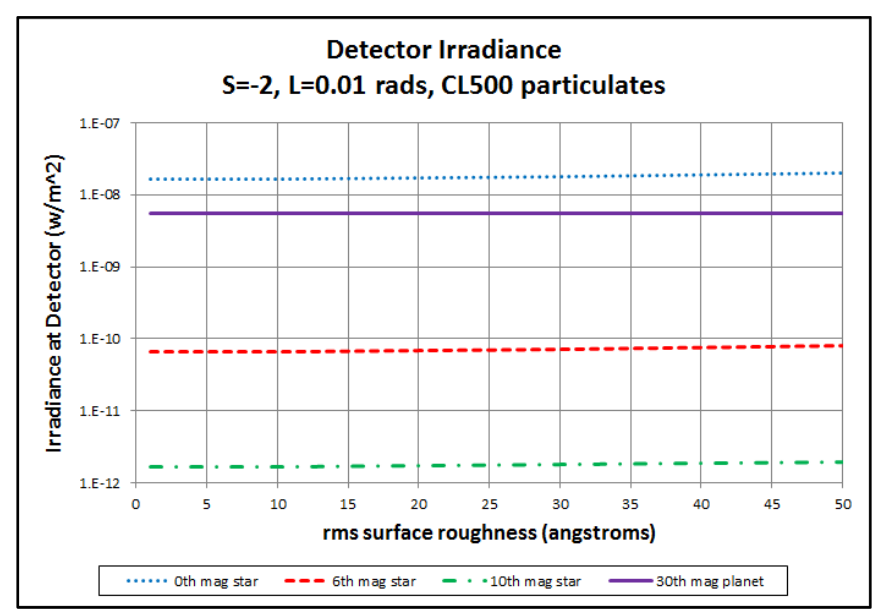

Figure 3 Detector irradiances for typical mirror surfaces and high level of particulates (CL500, corresponding to "end of life" [EOL] 5 years in orbit).

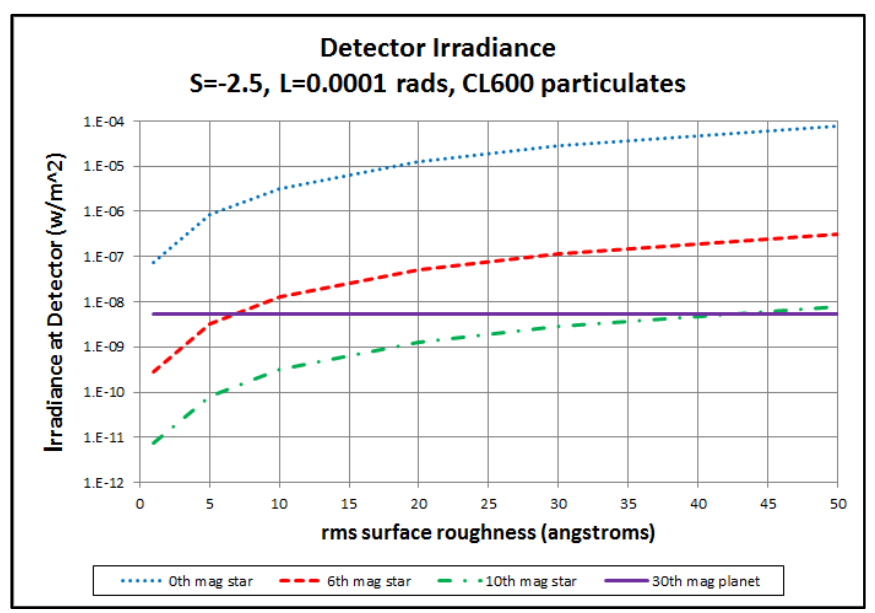

Figure 4 Detector irradiances for computer ground/polished mirror surfaces with high near-specular scatter and high level of particulates (comparable to James Webb Space Telescope [JWST], BOL, on orbit). 
Figure 7 shows the detector irradiances for a system employing conventionally polished mirrors $(\mathrm{S}=-2, \mathrm{~L}=0.01$ radians) contaminated with CL500 particulates corresponding to typical "end of life" (EOL) 5 years in orbit. As in the previous case, the planet is clearly visible against a $6^{\text {th }}$ or $10^{\text {th }}$ magnitude star, but now it is overwhelmed by the scatter produced by a $0^{\text {th }}$ magnitude star.

Figure 8 shows the detector irradiances for a system employing computer ground and polished mirrors (resulting in high near-specular scatter) contaminated with CL600 particulates comparable to the level of contamination expected in the James Webb Space Telescope, BOL, on orbit. Since the slope of the scatter function is so high $(\mathrm{S}=-2.9)$, there is a significant variation in the surface roughness scatter contribution. In this case, the planet is not discernable for a $0^{\text {th }}$ magnitude star, but becomes visible against a $10^{\text {th }}$ magnitude star over most of the range of plotted surface roughnesses. Against a $6^{\text {th }}$ magnitude star, the specific surface roughness determines planetary visibility.

\section{CONCLUSIONS}

Using reasonably simple expressions, it is possible to derive the scatter contributions from both mirror microroughness and particulate scatter at the detector of a Cassegrain telescope and then to compare these stray light levels to the imaged irradiance of a dim planet orbiting a star.

Although there are literally an infinite number of parametric possibilities, it is easy to come up with scenarios where the planet is visible. Likewise it is equally possible to generate scenarios where the planet is hopelessly overwhelmed by the scatter effects.

In order to construct a successful planet-finder telescope, many aspects of the system must be carefully considered:

- Basic optical configuration.

- Processing of the surfaces to minimize deleterious scatter contributions.

- Cleanliness of the surfaces in use.

- Relative brightnesses of the star and planet.

Since it is a coherent process, diffraction can be a more significant stray light problem than either surface roughness or particulate contamination.

\section{REFERENCES}

[1] Harvey, J., "Light Scattering Characteristics of Optical Surfaces “, Ph.D. dissertation, University of Arizona (1976).

[2] Institute of Environmental Sciences and Technology, www.iest.org.

[3] Peterson, R., Magallenes, P., Rock, D., "Tailored particle distributions derived from MIL-STD-1246”, Proc. SPIE 4774 (2002).

[4] Jenkins, D. G., Fest, E. C., Kremer, R. M., Spyak, P. R., "Improved Mie Theory Scatter Model for Particulate Contamination that Conserves Energy and Obeys Reciprocity", Proc. SPIE 6291 (2006).

[5] Van de Hulst, H., [Light Scattering by Small Particles], Dover (1957). 